\title{
Optimal Sensor Placement for a Truss Structure Using Particle Swarm Optimisation Algorithm
}

\author{
Jianhua Zhao and Xiaohong Wu \\ State Key Laboratory for Strength and Vibration of Mechanical Structures, School of Aerospace, Xi'an Jiaotong \\ University, Xi'an 710049, China.
}

\begin{abstract}
Qing Sun
Department of Civil Engineering, Xi'an Jiaotong University, Xi'an 710049, China, State Key Laboratory for Strength and Vibration of Mechanical Structures, School of Aerospace, Xi 'an Jiaotong University, Xi'an 710049, China.
\end{abstract}

\author{
Ling Zhang \\ State Key Laboratory for Strength and Vibration of Mechanical Structures, School of Aerospace, Xi'an Jiaotong \\ University, Xi'an 710049, China.
}

(Received 29 December 2014; accepted 13 April 2016)

\begin{abstract}
The problem of placing sensors plays a significant role in the domains of structural health monitoring (SHM) applications and parameter estimation in structural dynamics. In this paper, the particle swarm optimisation (PSO) algorithm is introduced firstly and utilised to place sensors optimally on a truss structure for the purpose of modal identification. Then, two different types of fitness functions are constructed as to be the optimal criteria, which are based on modal assurance criterion (MAC) and maximising measures of the observability gramian matrix, respectively. The former one is mainly used to distinguish between two sets of mode shapes with the aim of maximising the off-diagonal elements in MAC matrix, and the latter one ensures proper observability of the structure. Finally, a truss structure model is considered as an example to demonstrate the efficiency and validity of the proposed method. Numerical results show that the two approaches using the PSO algorithm can find the optimal location of sensors successfully and identify the modal frequencies of the truss structure accurately by acceleration FRF method.
\end{abstract}

\section{INTRODUCTION}

During the past several decades, a large number of research results have been reported on the structural health monitoring (SHM) and damage identification using modal data, such as large-scale space structures, high-rise buildings, long-span bridges and offshore platforms, etc. Therefore, the modal parameters identification from vibration data is crucial to estimate the state of structure in engineering domain. It is well known that the dynamic characteristics of a structure that can be extracted from measurements depend on the number and location of sensors. Inevitably, an important issue of the optimal sensor placement (OSP) should be encountered during pretest planning. Generally speaking, the more number of sensors are utilised, the more detailed information is obtained. The number of sensors applied in an engineering structure, however, is normally limited by the constraints of economic factors, workloads and placing space, etc. Hence, the sensors are placed in the most advantageous locations so as to achieve the best performance.

For the problem of optimal sensor placement, a variety of approaches have been developed on dealing with the optimal sensors placement problem, and detailed reports are published in literature. Early in 1991, Kammer ${ }^{1}$ developed an effective independence (EI) method for the selection of sensor locations from a larger candidate set based on their contribution to the determinant of the corresponding Fisher Information Matrix (FIM). Shih et al. ${ }^{2}$ proposed a method of placing sensors and actuators to obtain reliable measured data for estimating the modal parameters, which is based on the controllability and observability measures for second-order linear systems. Debnath et al. ${ }^{3}$ proposed the modal contribution in output energy (MCOE) which is evaluated using observability gramian as modal measure to evaluate modal participation and apply in this modal approach framework for sensor placement. Chow et al. ${ }^{4}$ addressed the problem of optimally placing sensors on a typical transmission tower so as to extract as much information as possible for structural model updating. Heo et al. ${ }^{5}$ presented a kinetic energy optimisation technique (EOT) to place sensors for SHM of a long span bridge. Cobb et al. ${ }^{6}$ and Shi et al. ${ }^{7}$ presented a method of optimising sensor locations for the purpose of localising structural damage sites. $\mathrm{Xu}$ et al. ${ }^{8}$ studied the optimisation of sensor locations based on the sensitivity analysis of the strain mode perturbation matrix with aim of damage detection for space truss structures. Papadimitriou ${ }^{9,10}$ proposed a rigorous formulation of the optimal sensor placement problem for structural identification based on the information entropy measure of parameter uncertainty. Meo et al. ${ }^{11}$ investigated six different optimal sensor placement techniques on a bridge structure with the aim of maximising the data information so that the structural dynamic behaviour can be fully characterised. Hanis et al. ${ }^{12}$ presented a new approach to op- 
timal placement of sensors in mechanical structures based on the effective independent (EI) method, and modified the criterion to meet the requirements of maximising useful signals and minimising spillover of unwanted modes at the same time.

In recent years, intelligent algorithms have received considerable attention for solving the problem of optimising the sensor placement, such as particle swarm optimisation (PSO), monkey algorithm (MA), simulated annealing (SA), genetic algorithm (GA), and so on. Rao et al. ${ }^{13}$ put forward to a hybrid PSO algorithm by combining a self-configurable PSO for arriving at an optimal number as well as locations of sensors on civil engineering structures for structural system identification and health monitoring. Yi et al. ${ }^{14}$ outlined the modified MA for optimal sensor placement in SHM of a high-rise building. Worden et al. ${ }^{15}$ made a review of OSP in SHM and applied the GA and the SA to determine the optimal sensor placement for fault diagnosis. Besides, GA has been widely applied to solve the optimal sensor placement problems due to their ability to solve large complex optimisation problems, which other methods have difficulty solving. For example, with the aim of modal identification, Yao et al. ${ }^{16}$ had taken advantage of the GA to place sensors optimally on a large space structure based on the maximising determinant of the FIM. Abdullah et al. ${ }^{17}$ utilised the GA in conjunction with gradient-based optimisation techniques to place sensors and actuators simultaneously in an effective structural control system. Guo et al. ${ }^{18}$ and Liu et al. ${ }^{19}$ developed an improved GA to find the optimal placement of sensors on a spatial lattice structure with the aim of SHM and detecting structural damage. Although abundance of literature has been published on the optimal placement of sensors, the novel and effective approaches are desirable to deal with the practical application.

This paper presents an optimal sensor placement method using the PSO algorithm for the purpose of obtaining reliable measured data and identifying the modal parameters. The paper is mainly focused on three aspects: (I) Two types of fitness functions are developed as the optimal criteria to find the best sensor locations, which are based on modal assurance criterion (MAC) and maximising measures of the observability gramian matrix, respectively; (II) the PSO algorithm is employed to solve the problem of OSP and determine the optimal location of sensors in the truss structure; and (III) the acceleration frequency response function (FRF) method is adopted to identify the modal parameters. The outline of this paper is organised as follows: Section 2 briefly introduces the basic theory of PSO algorithms. In section 3 , two different fitness functions are developed to determine the best sensor locations. The performance of the PSO scheme is verified by a numerical example for OSP in section 4. Finally, the overall conclusions of this paper are drawn in section 5 .

\section{PARTICLE SWARM OPTIMISATION (PSO) ALGORITHMS}

The particle swarm optimisation (PSO) is an efficient evolutionary computation technique, first proposed by Kennedy and Eberhart ${ }^{20}$ in 1995. As a category of swarm intelligence, the PSO is derived from the simulation of social behaviour of bird flocking or fish schooling during their search for food. Similarly to other evolutionary algorithms, the PSO is also a population-based swarm algorithm, which is used to deal with the global optimisation problems. In the standard PSO algorithm, all the members of population are called a swarm, and each individual within the swarm is regarded as a particle without mass and volume. During the process of searching for the best solutions, every particle is initialised with a random position and velocity in the search space, respectively, and each particle position represents a potential solution to the objective function. All the position and velocity of the particles are usually updated synchronously in the iteration of calculation. For details, every particle adjusts the velocity dynamically according to its own flying memory and its neighbour's experience. At the same time, the particle moves its position in the search domain toward a better position which is evaluated through a fitness value.

Assuming that there is a $m$-dimensional search space, and the swarm is composed of $n$ particles. The position and velocity vectors of the $i$ th particle can be denoted as $X_{i}=$ $\left(x_{i 1}, x_{i 2}, \cdots, x_{i m}\right)$ and $V_{i}=\left(v_{i 1}, v_{i 2}, \cdots, v_{i m}\right), i=1,2$, $\cdots, n$, respectively. Meanwhile, the particle's velocity is restricted within a range of $\left[-v_{\max }, v_{\max }\right.$ to avoid missing the effective solutions during the particle's flying. In an iterative manner, two extreme values are used to modify the particle's position according to the best fitness it has achieved at present. The first one is the personal best position of particle $i$ until the current iteration, called pbest, which can be represented as $P_{i}=\left(p_{i 1}, p_{i 2}, \cdots, p_{i m}\right)$. The other is the global best position of the whole swarm obtained so far, named gbest, which is denoted by $P_{g}=\left(p_{g 1}, p_{g 2}, \cdots, p_{g m}\right)$. Numerically, the updated velocity and position of each particle can be written as

$$
\begin{gathered}
V_{i}(t+1)=\omega V_{i}(t)+c_{1} r_{1}\left(P_{i}(t)-X_{i}(t)\right) \\
+c_{2} r_{2}\left(P_{g}(t)-X_{i}(t)\right) ; \\
X_{i}(t+1)=X_{i}(t)+V_{i}(t+1) ;
\end{gathered}
$$

where $t$ is the iteration index; parameter $\omega$ is the inertia weight, which is used to accelerate the convergence and balance the global and local search abilities of the particles; $c_{1}$ and $c_{2}$ are the acceleration coefficients, controlling the relative influence of the particle velocity towards pbest and gbest, respectively; $r_{1}$ and $r_{2}$ are two uniformly distributed random numbers between 0 and 1 . The updating process and the movement of a particle are illustrated in Fig. 1. Based on the theory of PSO as introduced above, the main steps can be described as follows:

- Step 1. Set the basic parameters, including the number of particles, the number of iterations (generations), acceleration coefficients $c_{1}$ and $c_{2}$, random numbers $r_{1}$ and $r_{2}$;

- Step 2. Initialise the positions and velocities of the particles randomly;

- Step 3. Evaluate the fitness value of each particle at its current position and then update the personal best position $P_{i}$ and the global best position $P_{g}$;

- Step 4. Update the new velocity according to Eq. (1) and obtain the new position of the particle in Eq. (2);

- Step 5. Repeat the steps 2 through 4 until the maximum number of iterations is reached. 


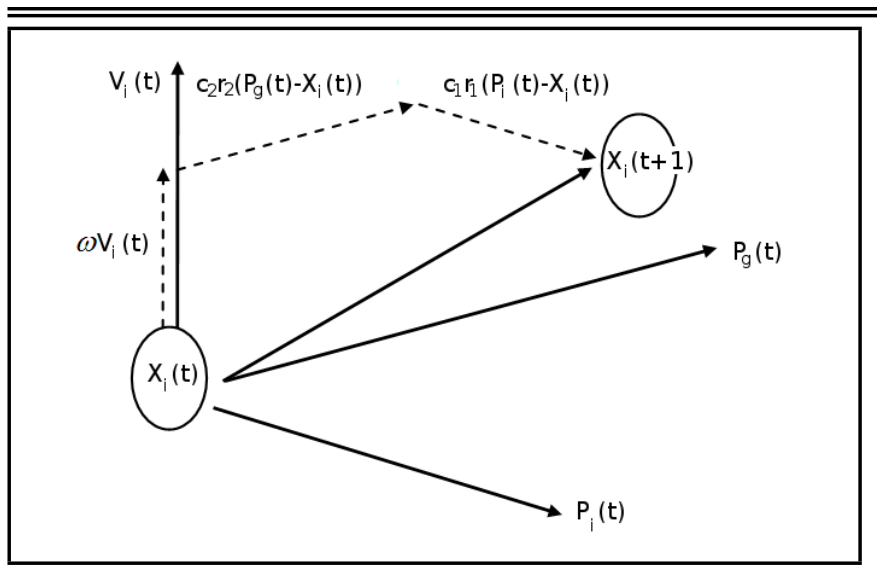

Figure 1. Particle's updating process of PSO in 2D space.

Table 1. The basic parameters in PSO.

\begin{tabular}{|c|c|}
\hline Parameters & Values \\
\hline Population sizes & $N=20$ \\
\hline Inertia weight & $w(t)=0.4$ \\
& $+0.5 *\left(T_{\max }-t\right) / T_{\max }$ \\
\hline $\begin{array}{c}\text { Acceleration coefficients of } \\
\text { particle velocity }\end{array}$ & $c_{1}=c_{2}=1.49445$ \\
\hline Maximum velocity & $v_{\max }=4$ \\
\hline Maximum number of iterations & $T_{\max }=200$ \\
\hline
\end{tabular}

To verify the efficiency and stability of the PSO algorithm, a test function called the Schaffer function is presented in this subsection. All the basic parameters in PSO are listed in Table 1. The Schaffer function is expressed as

$$
\begin{array}{r}
\max f(x, y)=0.5-\frac{\sin ^{2} \sqrt{x^{2}+y^{2}}-0.5}{\left[1.0+0.001\left(x^{2}+y^{2}\right)\right]^{2}}, \\
x, y \in(-4,4) ;
\end{array}
$$

which has only one global maximum: $(0,0)$ and $f\left(x^{*}, y^{*}\right)=$ 1. The plot of Schaffer function is shown in Fig. 2. As depicted in Fig. 2, it is clearly that the maximal value is at the centre peak.

In the simulation of the PSO process, the particle population is randomly initialised by uniformly sampling in the feasible solution space for the test function. It is worth noticing that the results obtained from a PSO process with the limited number of iterations might be a sub-optimal solution. To get a result with higher confidence, one must run the PSO process several times, each with a randomly generated initial condition. In order to obtain more accurate results, 10 runs are completed

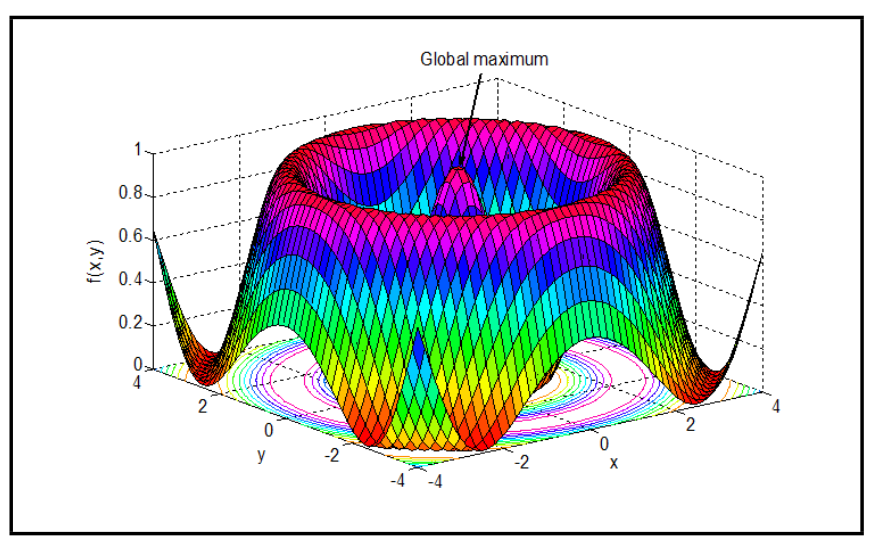

Figure 2. Plot of the test function.

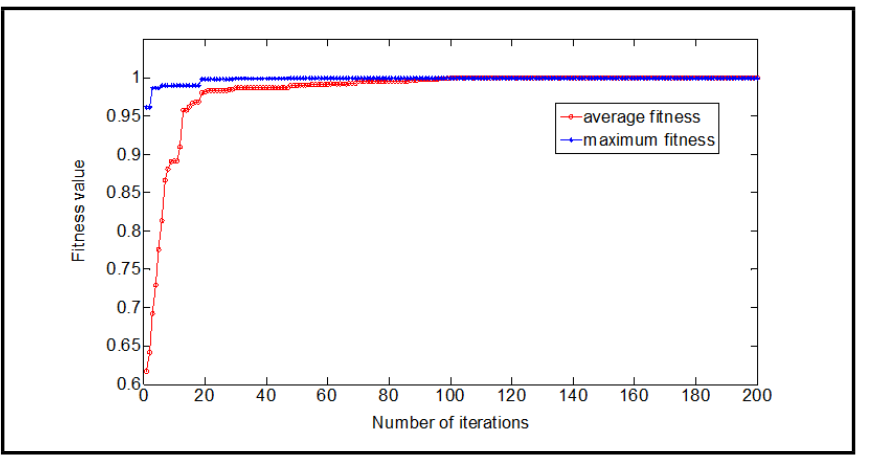

Figure 3. Converge curves of the test function.

for the Schaffer function. The optimal results are presented in Table 2 and a typical convergence curve of the test function is plotted in Fig. 3. The optimal results in the last row of Table 2 are the means of the corresponding values. The average number of iterations is about 155, and the optimal test function value is 1 . As can be seen in Fig. 3, it is shown the sound stability and convergence in the values of test function plots.

\section{FITNESS FUNCTION OF THE OPTIMAL SENSOR LOCATION}

To determine the optimal sensors location of a typical structure and identify the modal parameters, a fitness function, or performance function as it is commonly called is required. The main purpose here is to validate the PSO-based optimal sensor placement algorithm by evaluating the corresponding fitness function. In this section, two types of fitness functions are constructed, namely, the modal assurance criterion (MAC) and the observability gramian matrix, respectively. The first one considered is maximisation of the value of all the off-diagonal elements in MAC matrix and the virtue of this fitness is that the size of the off-diagonal elements can be regarded as an indication of the fitness to place the sensors. The second one aims to maximize the observability gramian matrix, which ensures proper observability of each mode considering them with homogeneity.

\subsection{Modal Assurance Criterion Based Method}

For the purpose of the vibration measurement of structures, the mode shapes and structural dynamic response under external forces are concerned due to the significant role they have played in the process of structural analysis. Hence, the sensors should be placed in the positions where the vibration amplitudes or where the changes of the vibration mode shape are relatively large. ${ }^{21}$ The MAC is probably the most commonly employed to indicate the correlation between two sets of mode shapes and is defined as follows ${ }^{22}$

$$
M A C_{i j}=\frac{\left|\varphi_{i}^{T} \varphi_{j}\right|^{2}}{\left(\varphi_{i}^{T} \varphi_{i}\right)\left(\varphi_{j}^{T} \varphi_{j}\right)}
$$

in which $\varphi_{i}$ and $\varphi_{j}$ are the $i$ th mode shape and the $j$ th mode shape of the same measurement point, respectively. The value of $M A C$ is limited to 0 and 1 . When the two sets of mode shapes fit each other well, the value is close to 1 . Conversely, a value of 0 indicates uncorrelated (orthogonal) vectors or no 
Table 2. Optimisation results of Schaffer function from ten runs.

\begin{tabular}{|c|c|c|c|c|c|}
\hline \multirow[t]{2}{*}{ No. } & \multicolumn{2}{|c|}{ the global optimum $P_{g d}$} & \multirow{2}{*}{$\begin{array}{l}\text { Maximum fitness } \\
\text { value } f_{\max }\end{array}$} & \multirow[t]{2}{*}{ No. of iterations } & \multirow[t]{2}{*}{ Running time/s } \\
\hline & $x$ & $y$ & & & \\
\hline 1 & $-1.866 \mathrm{e}-9$ & $-9.155 e-10$ & 1 & 158 & 0.94 \\
\hline 2 & $5.797 \mathrm{e}-10$ & $-2.954 \mathrm{e}-9$ & 1 & 149 & 0.93 \\
\hline 3 & $-4.724 \mathrm{e}-9$ & $-2.449 \mathrm{e}-9$ & 1 & 141 & 0.92 \\
\hline 4 & $-4.988 \mathrm{e}-9$ & $-3.418 \mathrm{e}-9$ & 1 & 154 & 0.96 \\
\hline 5 & $-4.527 \mathrm{e}-10$ & $1.891 \mathrm{e}-9$ & 1 & 156 & 0.94 \\
\hline 6 & $-3.749 \mathrm{e}-9$ & $2.662 \mathrm{e}-9$ & 1 & 157 & 0.93 \\
\hline 7 & $8.545 \mathrm{e}-10$ & $-4.509 \mathrm{e}-9$ & 1 & 151 & 0.93 \\
\hline 8 & $-7.27 e-9$ & $-1.509 \mathrm{e}-9$ & 1 & 160 & 0.96 \\
\hline 9 & $-2.197 \mathrm{e}-9$ & $2.863 \mathrm{e}-9$ & 1 & 155 & 0.93 \\
\hline 10 & $7.786 \mathrm{e}-9$ & $-1.258 \mathrm{e}-8$ & 1 & 165 & 0.94 \\
\hline Average & $-5.098 \mathrm{e}-8$ & $-3.593 e-8$ & 1 & 154.6 & 0.94 \\
\hline
\end{tabular}

correlation between the off-diagonal elements $M A C_{i j}(i \neq j)$. where

The superscript $T$ indicates the transpose of the vectors.

The fitness function can be formulated using the MAC as follows

$$
f_{1}=1-\max _{i \neq j}\left\{a b s\left(M A C_{i j}\right)\right\} ;
$$

where $\operatorname{abs}(\cdot)$ indicates the absolute value and $\max (\cdot)$ denotes the maximal value. The greatest benefit of choosing this fitness function is that the comparison of the two sets of mode shapes does not require any mass and stiffness information of the structure. Maximising the fitness function value will guarantee to find the best sensor locations where the mode shapes have the largest change between the different orders.

\subsection{Maximisation Method}

Observability-Based

In order to acquire proper observability of each mode considering them with homogeneity and not globally, as it is usually done, any measure of the observability of a dynamic system should reflect the amount of information that can be derived about the system states from the sensor outputs in the presence of measurement noise. ${ }^{23}$ In other words, the sensor locations should produce as much as possible information about the contributions of individual modes to the system response.

When the system is released from the initial state $x(0)=x$, the maximum output energy received by the sensors is given by

$$
J=\int_{0}^{\infty} y^{T}(t) y(t) d t=x_{o}^{T} W_{o} x_{o} ;
$$

where $W_{o}$ is the observability gramian matrix and defined as

$$
W_{o}=\int_{0}^{\infty} \exp \left(A^{T} t\right) C^{T} C \exp (A t) d t .
$$

The exact (full) observability gramian can be obtained from the Lyapunov equation

$$
A^{T} W_{o}+W_{o} A+C^{T} C=0 .
$$

Assume the total number of modes in the model is $N$. For the first $i$ th modes $(i=1, \cdots, N)$, the Eq. (8) can be rewritten in a simplified form

$$
w_{o i}\left(A_{i}+A_{i}^{T}\right)+C_{i}^{T} C_{i}=0
$$

$$
\begin{aligned}
& A_{i}=\left[\begin{array}{cc}
0 & \omega_{i} \\
-\omega_{i} & -2 \zeta_{i} \omega_{i}
\end{array}\right] ; \\
& C_{i}^{T} C_{i}=\left[\begin{array}{cc}
0 & 0 \\
0 & \left\|C_{i}\right\|_{2}^{2}
\end{array}\right] .
\end{aligned}
$$

Substituting $A_{i}$ and $C_{i}^{T} C_{i}$ into Eq. (9), the observability gramian for the $i$ th mode is

$$
w_{o i}=\frac{\left\|C_{i}\right\|_{2}^{2}}{4 \zeta_{i} \omega_{i}} ;
$$

in which, $C_{i}$ is the $i$ th mode "s contribution to the output matrix and $\|X\|_{2}$ represents the Euclidean norm of $X$.

As described above, it can be shown that the observability gramian depends on matrix $C$ that is associated with the sensors location. Information about observability is hidden in eigenvalues of the observability gramian matrix. If the $i$ th eigenvalue of $W$ is small, it means that the $i$ th mode will not be well observed. To avoid this case and find the optimal locations of sensors, a new fitness function by a measure of $W$ is given as

$$
f_{2}=\max \left\{\operatorname{trace}\left(W_{o}\right) * \sqrt[2 n]{\operatorname{det} W_{o}} / \sigma\left(\lambda_{i}\right)\right\} ;
$$

where $n$ is the number of the first modes to be observed; $\operatorname{trace}\left(W_{o}\right)$ denotes the trace of the matrix $W_{o}$, namely, the sum of eigenvalues of the matrix $W_{o} ; \sigma\left(\lambda_{i}\right)$ is the standard deviation of the gramian eigenvalues $\lambda_{i}$; $\sqrt[2 n]{\operatorname{det} W_{o}}$ represents the geometric mean of eigenvalues. This fitness function ensures global observability of the system for the first $n$ eigenmodes and the aim here is desirable to find the best sensors location in such a way as to maximise eigenvalues of the observability gramian matrix.

\section{NUMERICAL EXAMPLE}

To illustrate the usefulness of the proposed approach, two different optimal sensor placement strategies are performed using a 10-bay plain truss model. The truss is clamped at both ends and modelled by a finite element beam model as shown in Fig. 4. The finite model of the truss consists of 20 nodes and 37 elements. Each node has two translation degrees of freedom (DOF). The material properties and geometric parameters of the structures are as follows: elastic modulus $E=210 \mathrm{GPa}$, Poisson's ratio $\eta=0.3$, mass density $\rho=7.8 \times 10^{3} \mathrm{Kg} / \mathrm{m}^{3}$, and the length of each element $l=0.5 \mathrm{~m}$. All the element cross-sections are circular tubes and the dimensions are $\Phi 16 \mathrm{~mm} \times 2 \mathrm{~mm}$. The deadweight of all the rods 


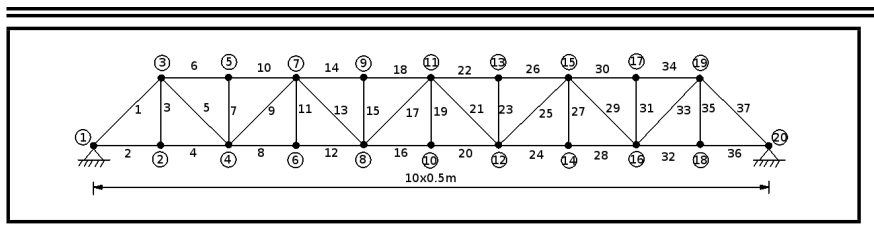

Figure 4. A 10-bay plain truss modal.

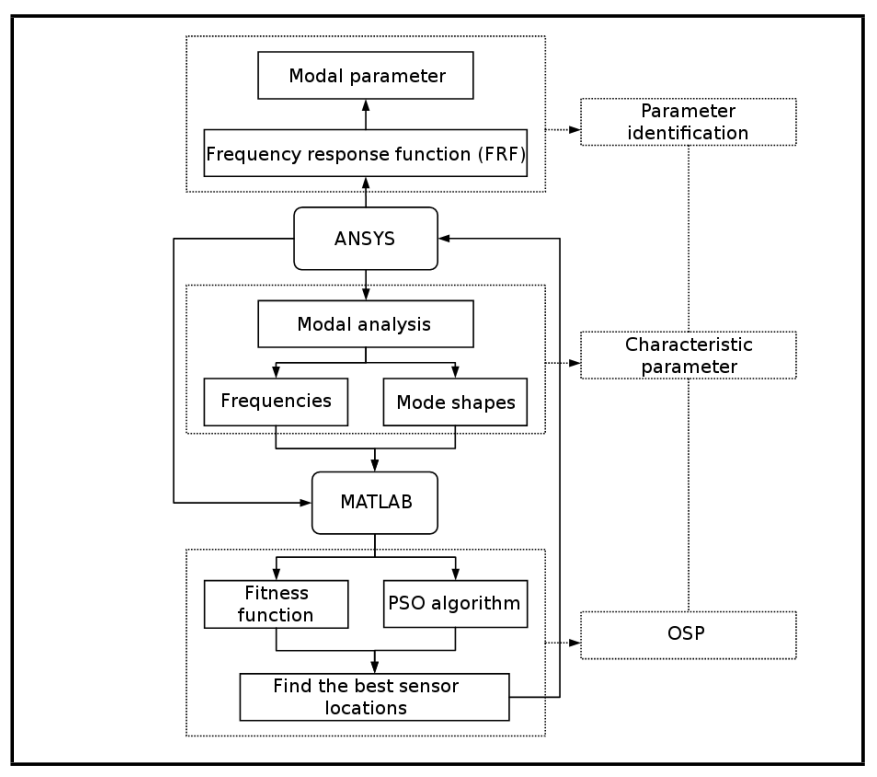

Figure 5. The block diagram of calculation process.

and a $3 \mathrm{Kg}$ ball are regarded as lumped mass concentrated at the nodes. The truss model is generated by the commercial finite element analysis software ANSYS. The spatial (mass and stiffness) and modal (eigenvalues and eigenvectors or named natural frequencies and mass-normalised mode shapes) matrices calculated with ANSYS are exported to MATLAB for use with the PSO algorithm develop for this project. The block diagram of calculation process is shown in Fig. 5. According to the practical experience and engineering judgement, each measurement point is merely considered the vertical displacement of translation motion. Therefore, there are 18 unclamped nodes which are selected as the candidate location to place sensors. Here, suppose 2, 4 and 6 sensors need to be placed on the truss structure to identify the target modes, respectively.

The first six natural frequencies and mode shapes are selected as the target modes for determining the sensor locations, which are shown in Table 3 and Fig. 6. Two different sensor placement approaches, which correspond to the two types of fitness functions $\left(f_{1}\right.$ and $\left.f_{2}\right)$ mentioned in section 3 , will be implemented to find the best sensor locations using the PSO algorithm. The basic parameters of PSO used in the analysis are listed as follows: maximum velocity is 0.5 , maximum number of iterations is 300 , and the other parameters are identical with those in Table 1. Each of approaches is applied to select the best sensor locations for identification of the six target modes in the 10-bay plain truss modal. The main objective here is to determine the best location of sensors where the structural dynamic characteristics and the structural dynamic response data of 10-bay plain truss modal can be picked up completely. All the optimal results for 2, 4, and 6 sensor locations are calculated by using PSO algorithm and listed in Table 4 . It is observed that sensors have been placed in the symmetrical and

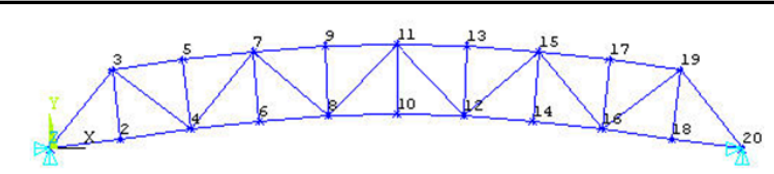

a) the 1 st mode

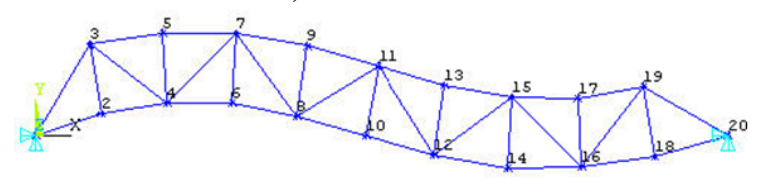

b) the 2 nd mode

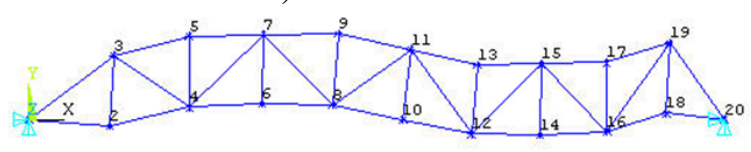

c) the $3 \mathrm{rd}$ mode

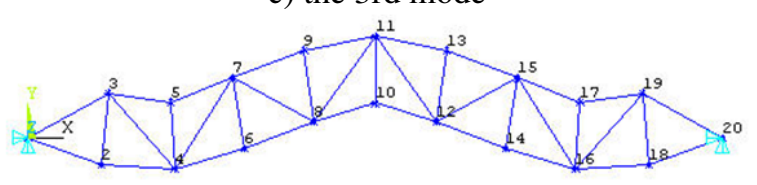

d) the 4th mode

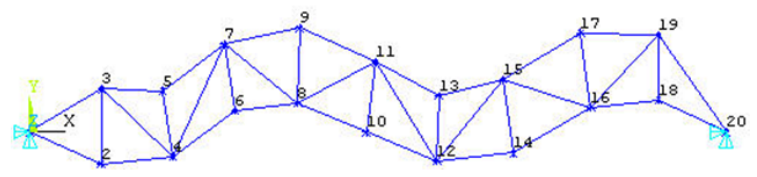

e) the 5 th mode

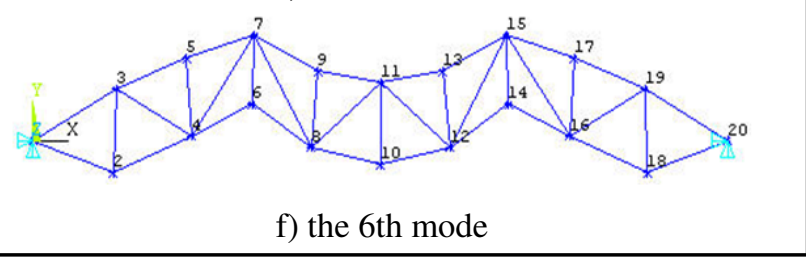

Figure 6. The first six mode shapes of the truss model.

anti-symmetrical positions or near to these positions, which are similar to the previous results reported. ${ }^{19}$ The primary reason is that the truss model and constraints are symmetrical. Further to verify the effectiveness and robustness of the proposed method in this paper, the PSO process of optimising sensors placement has been run for 10 times each with a stochastic initial population. Numbers of the convergence iterations and the maximum fitness values are compared in Table 5, where it can be observed that the maximum fitness values tend to a stable constant in all cases. That means the proposed method is valid in solving the problem of OSP.

To evaluate the stability and reliability of the PSO process, all the typical fitness convergence curves of the two fitness functions with different number of sensors (measured points) are depicted in Figs. 7-9. Figures 7(a1), 8(b1), and 9(c1) show that with the fitness function $f_{1}$ the convergence curves reach their maximum fitness values at the less than $300^{\text {th }}$ iterations. From Figs. 7(a1), 8(b1), and 9(c1), it can be observed that all the maximum fitness values using PSO tend to a constant quickly and the average fitness value steadily tends to maximum fitness value along with the increasing number of iterations. Obviously, the convergence characteristics are very well in the iterative process. In other words, the results show the sound stability and convergence in the fitness function plots. Compared Figs. 7(a1), 8(b1), and 9(c1), it can also be observed 
Table 3. The first six natural frequencies and descriptions of mode shapes for the truss model.

\begin{tabular}{||c|c|c||}
\hline No. of Modes & Frequency/Hz & Description \\
\hline 1 & 28.55 & First symmetric bending deflection \\
\hline 2 & 71.94 & Second anti-symmetric bending deflection \\
\hline 3 & 132.69 & Third anti-symmetric bending deflection \\
\hline 4 & 138.40 & Fourth symmetric bending deflection \\
\hline 5 & 200.30 & Fifth anti-symmetric bending deflection \\
\hline 6 & 234.01 & Sixth symmetric bending deflection \\
\hline
\end{tabular}

Table 4. Sensor placement results of the truss structure.

\begin{tabular}{|c|c|c|}
\hline \multirow{2}{*}{ No. of sensors } & Fitness function $f_{1}$ & Fitness function $f_{2}$ \\
\cline { 2 - 3 } & Optimal sensor locations (No. of nodes) & Optimal sensor locations (No. of nodes) \\
\hline 2 & 7,17 & 9,18 \\
\hline 4 & $4,8,12,17$ & $2,9,16,18$ \\
\hline 6 & $2,6,7,10,15,18$ & $3,7,10,15,18,19$ \\
\hline
\end{tabular}

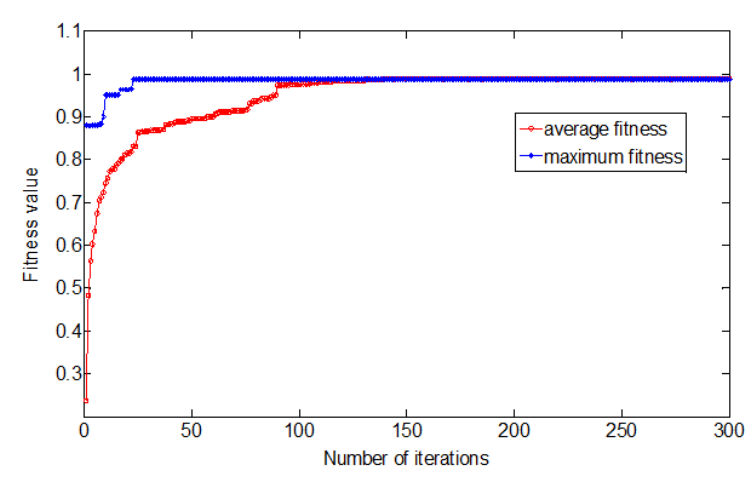

a1)

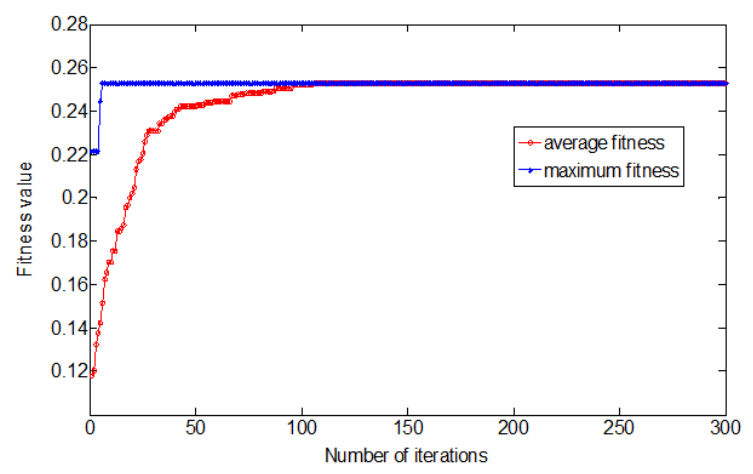

a2)

Figure 7. The typical fitness curves of PSO algorithm with different fitness function ( 2 sensors): (a1) $f_{1}$ and (a2) $f_{2}$.

that the fewer number of sensors are placed, the faster convergence speed will be acquired. The reason is that the more number of sensors will increase the complexity of the optimisation and waste plenty of computational time. As can be seen in Fig. 7(a2), 8(b2), and 9(c2), in which the fitness convergence curves are based on the fitness function $f_{2}$, similar conclusions can also be summarised as described above. As a consequence, the PSO consistently converges to the maximum fitness value in less iteration during the optimisation process. That is to say, the proposed method is effective and efficient in optimising the sensor locations of a truss structure.

Furthermore, for the sake of demonstrating the capability of capturing the dynamic characteristics of the 10-bay plain truss model using the two different optimal sensor placement techniques, the acceleration frequency response function (FRF) method is adopted to identify the modal frequencies for comparing with those computing by FEM. Here, the acceleration

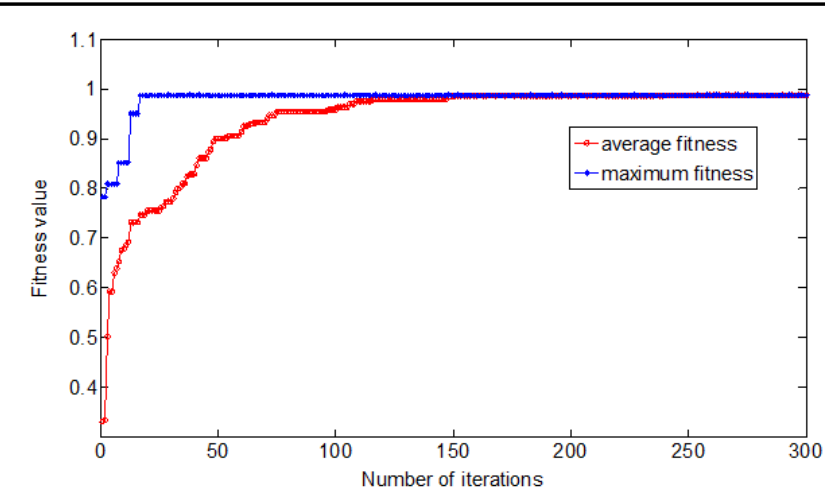

b1)

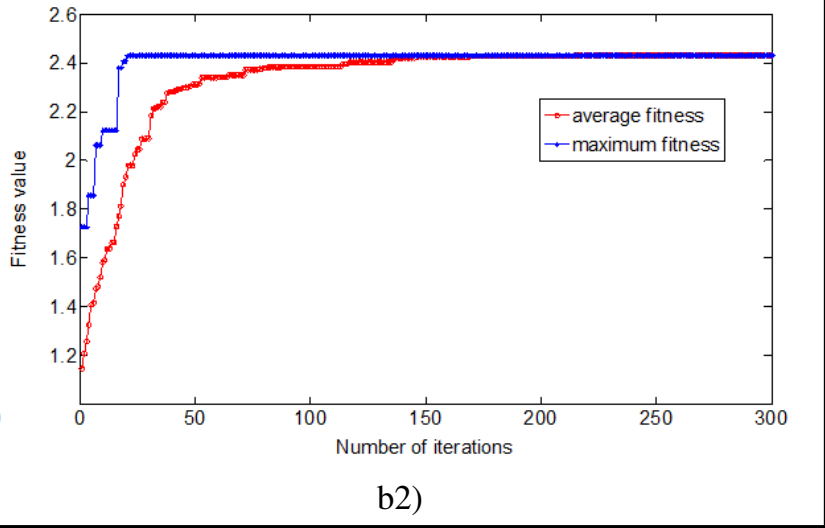

Figure 8. The typical fitness curves of PSO algorithm with different fitness function (4 sensors): (b1) $f_{1}$ and (b2) $f_{2}$.

frequency response can be expressed as follow ${ }^{24}$

$$
a_{p q}(\omega)=\sum_{j=1}^{n} \frac{-\omega^{2} \phi_{j p} \phi_{j p}}{\omega_{j}^{2}-\omega^{2}+2 i \xi_{j} \omega \omega_{j}}
$$

where $i=\sqrt{-1}$ denotes the imaginary unit; $\omega$ represents the forcing frequency in $\mathrm{rad} / \mathrm{s} ; \omega_{j}$ and $\xi_{j}$ are the undamped natural frequency of the $j$ th mode and the damping coefficient, respectively; $\phi_{j q}$ is the $j$ th mode shape at the $q$ th input location, and $\phi_{j p}$ is the $j$ th mode shape at the $p$ th output location.

Then, the frequency response data matrix $A$ can be defined as a collection of the individual frequency response matrices from the desired frequency band

$$
A=\left[\begin{array}{lllll}
a\left(\omega_{1}\right) & a\left(\omega_{2}\right) & a\left(\omega_{3}\right) & \cdots & a\left(\omega_{m}\right)
\end{array}\right]
$$

in which $m$ is the number of data points, and $a\left(\omega_{i}\right) \in$ $C^{m_{s} \times m_{a}}$, where $m_{s}$ and $m_{a}$ are the number of sensors and inputs, respectively. 
Table 5. Comparison of the convergence and maximum fitness values by using different fitness functions $\left(f_{1}\right.$ and $\left.f_{2}\right)$.

\begin{tabular}{|c|c|c|c|c|c|c|c|c|c|c|c|c|}
\hline \multirow{3}{*}{ No. } & \multicolumn{6}{|c|}{ Fitness function $f_{1}$} & \multicolumn{6}{|c|}{ Fitness function $f_{2}$} \\
\hline & \multicolumn{2}{|c|}{2 sensors } & \multicolumn{2}{|c|}{4 sensors } & \multicolumn{2}{|c|}{6 sensors } & \multicolumn{2}{|c|}{2 sensors } & \multicolumn{2}{|c|}{4 sensors } & \multicolumn{2}{|c|}{6 sensors } \\
\hline & $\begin{array}{c}\text { No. of } \\
\text { iterations }\end{array}$ & $\begin{array}{c}\text { Fitness } \\
\text { values }\end{array}$ & $\begin{array}{c}\text { No. of } \\
\text { iterations }\end{array}$ & $\begin{array}{c}\text { Fitness } \\
\text { values }\end{array}$ & $\begin{array}{c}\text { No. of } \\
\text { iterations }\end{array}$ & $\begin{array}{c}\text { Fitness } \\
\text { values }\end{array}$ & $\begin{array}{c}\text { No. of } \\
\text { iterations }\end{array}$ & $\begin{array}{c}\text { Fitness } \\
\text { values }\end{array}$ & $\begin{array}{c}\text { No. of } \\
\text { iterations }\end{array}$ & $\begin{array}{c}\text { Fitness } \\
\text { values }\end{array}$ & $\begin{array}{c}\text { No. of } \\
\text { iterations }\end{array}$ & $\begin{array}{c}\text { Fitness } \\
\text { values }\end{array}$ \\
\hline 2 & 126 & 0.9876 & 135 & 0.9785 & 237 & 0.9778 & 119 & 0.2528 & 148 & 2.4321 & 176 & 4.2485 \\
\hline 3 & 134 & 0.9878 & 132 & 0.9673 & 232 & 0.9741 & 127 & 0.2527 & 143 & 2.4321 & 188 & 4.2503 \\
\hline 4 & 139 & 0.9878 & 128 & 0.9782 & 241 & 0.9778 & 113 & 0.2528 & 165 & 2.4321 & 192 & 4.2502 \\
\hline 6 & 118 & 0.9876 & 137 & 0.9685 & 223 & 0.9655 & 143 & 0.2527 & 139 & 2.4319 & 195 & 4.3079 \\
\hline 7 & 131 & 0.9876 & 134 & 0.9673 & 238 & 0.9761 & 121 & 0.2527 & 148 & 2.4321 & 183 & 4.2344 \\
\hline 8 & 127 & 0.9877 & 126 & 0.9782 & 248 & 0.9655 & 124 & 0.2527 & 156 & 2.4321 & 189 & 4.3079 \\
\hline 9 & 143 & 0.9876 & 141 & 0.9785 & 219 & 0.9778 & 131 & 0.2527 & 161 & 2.4321 & 194 & 4.3079 \\
\hline 10 & 124 & 0.9876 & 135 & 0.9785 & 236 & 0.9761 & 109 & 0.2527 & 155 & 2.4321 & 186 & 4.2503 \\
\hline Average & 129.6 & 0.9866 & 137.1 & 0.9752 & 232.7 & 0.9718 & 124.3 & 0.2527 & 152.5 & 2.4320 & 190.8 & 4.2773 \\
\hline
\end{tabular}

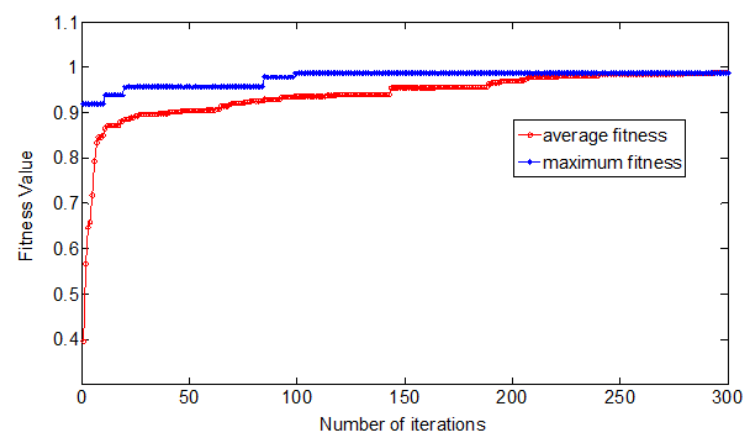

c1)

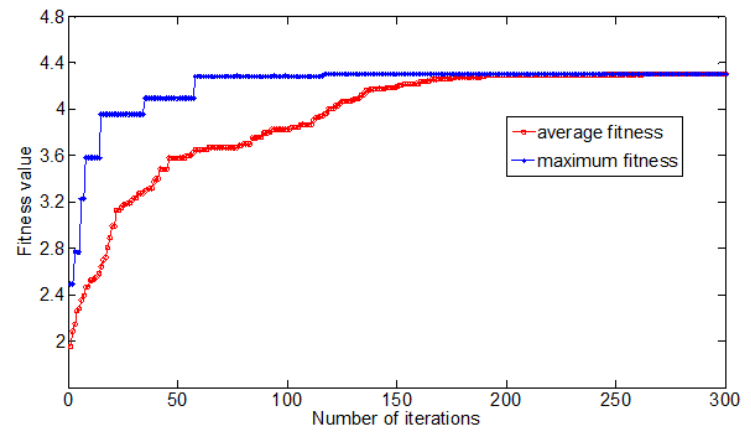

c2)

Figure 9. The typical fitness curves of PSO algorithm with different fitness function (6 sensors): (c1) $f_{1}$ and (c2) $f_{2}$.

For the purpose of identifying dynamic response of the structure, a step force excitation is applied on the vertical direction at node 11 of the model. This step force excitation can be regarded as simulating the excitation from impact hammer tests. In addition, this example is run with 1 percent modal damping in all the cases. The frequency response functions are calculated by using Eq. (14) with all considered mode shapes, and all the measured FRFs corresponding to the excitation location at the mid-point of the model and responses at different measured locations are depicted in Figs. 10-12, respectively. From the aforementioned results, it can be seen clearly that the first six frequencies can be identified rapidly. Specifically, it is noted that the peaks for all the first modes are very sharp in the figures, which implies that the first natural frequency is principal in the frequency domain. The peak corresponding to the third mode, however, is not very sharp in the plots of acceleration FRFs. The reason for this is that the third natural frequency is close to the fourth one, the amplitude of FRF cor- responding to the lower natural frequency is suppressed by the higher one. In theory, it is enough to extract the modal frequency of the structure by only one sensor. Nevertheless, to obtain the more reliable structural dynamic information, there are several sensors that are installed in practical engineering applications. In this work, we choose the average value of modal frequencies measured at different locations as the final identification result. The main purpose of doing so is to reduce the measurement errors and improve the accuracy of the modal identification. The results of modal frequencies identification are tabulated in Table 6. As a comprehensive comparison between Table 3 and Table 6, it is evident that the identified modal frequencies results are very close to those calculated by FEM and the maximum error is less than 2.3 percent, which satisfies the engineering requirements. This means that the modal frequencies of the structure can be identified accurately by FRF method. It is also noted that the higher modal frequencies are not identified so accurately as the lower ones, because the contribution of higher modes to the dynamic response of the structure is somewhat less than that of the lower modes. $^{25}$

\section{CONCLUSIONS}

This paper has investigated the problem of optimal sensor placement using the PSO algorithm for vibration testing and modal identification. In order to find the optimal location of sensors in the structure, two different types of fitness function have been employed for the optimisation problem. The first one is obtained by maximising the modal assurance criterion (MAC) and the second one is based on maximising a measure of the observability gramian matrix. PSO algorithms are well adapted to solve these optimisation problems of the fixed number of sensors. The feasibility and effectiveness of the proposed method are illustrated via an extensive numerical simulation studies on the modal identification of a 10-bay plain truss structure. The simulation results show the efficiency of PSO for these optimisation problems and indicate that the proposed method is capable of solving problem with potential candidate sensor locations well and identifying the modal frequencies of the truss structure accurately by acceleration FRF method. Future works will be devoted to validating the proposed method by experimentally testing for more complex structures. 


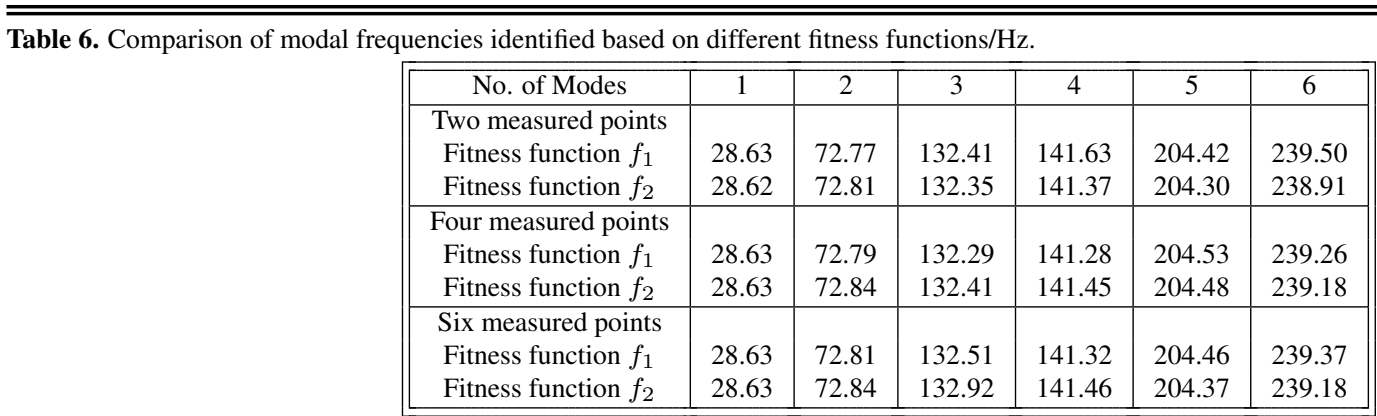

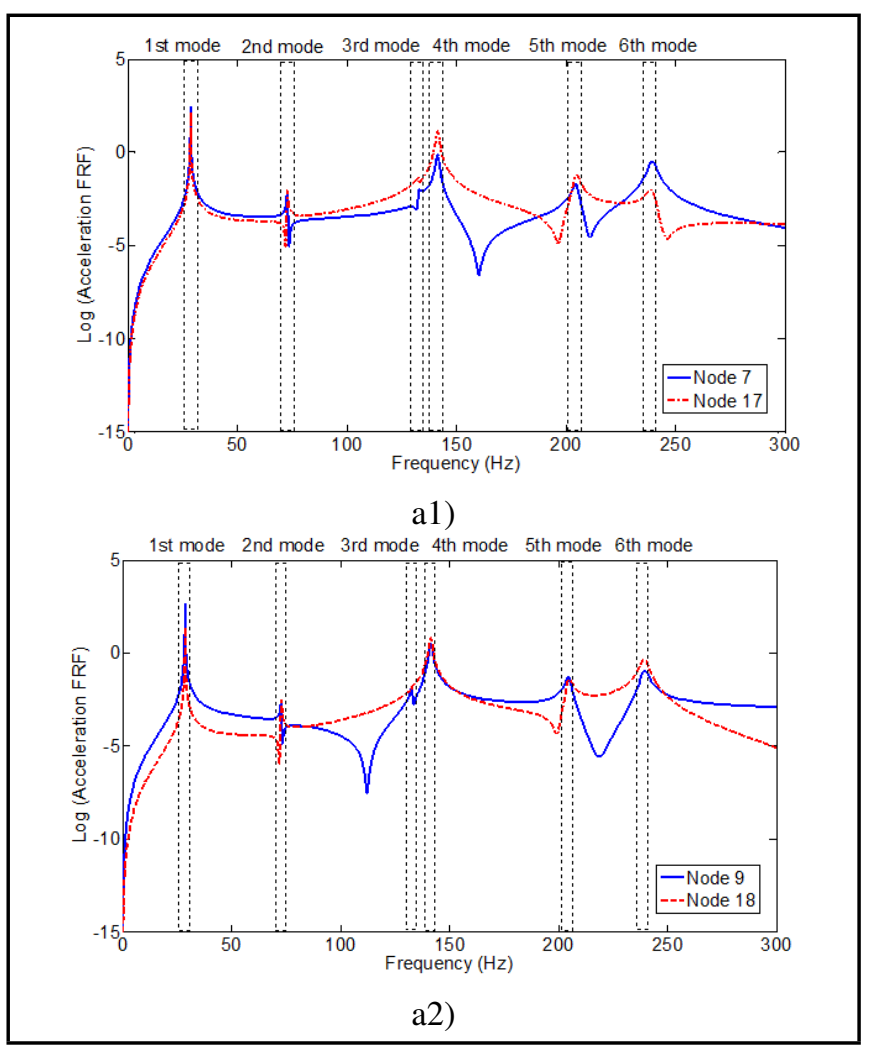

Figure 10. Comparisons of the FRF at different measured locations ( 2 sensors): (a1) measured locations determined based on fitness function $f_{1}$ and (a2) measured locations determined based on fitness function $f_{2}$.

\section{ACKNOWLEDGEMENTS}

This research is funded by the National Natural Science Foundation, People's Republic of China (grant No. 11172226). This support is gratefully acknowledged.

\section{REFERENCES}

1 Kammer, D. C. Sensor placement for on-orbit modal identification and correlation of large space structures, Journal of Guidance, Control and Dynamics, 14 (2), 251-259, (1991). https://dx.doi.org/10.2514/3.20635

2 Shih, Y. T., Lee, A. C., and Chen, J. H. Sensor and actuator placement for modal identification, Mechanical Systems and Signal Processing, 12 (5), 641-659, (1998). https://dx.doi.org/10.1006/mssp.1998.0168

3 Debnath, N., Dutta, A., and Deb, S. K. Placement of sensors in operational modal analysis for truss bridges, Mechanical Systems and Signal Processing, 31, 196-216, (2012). https://dx.doi.org/10.1016/j.ymssp.2012.04.006

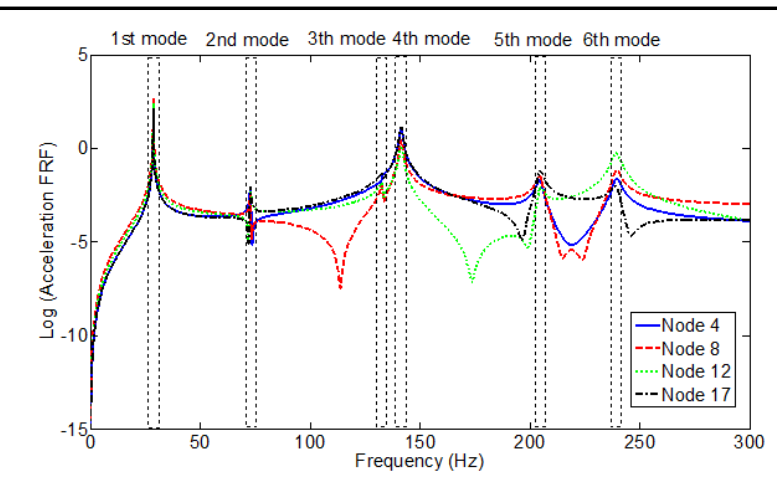

b1)

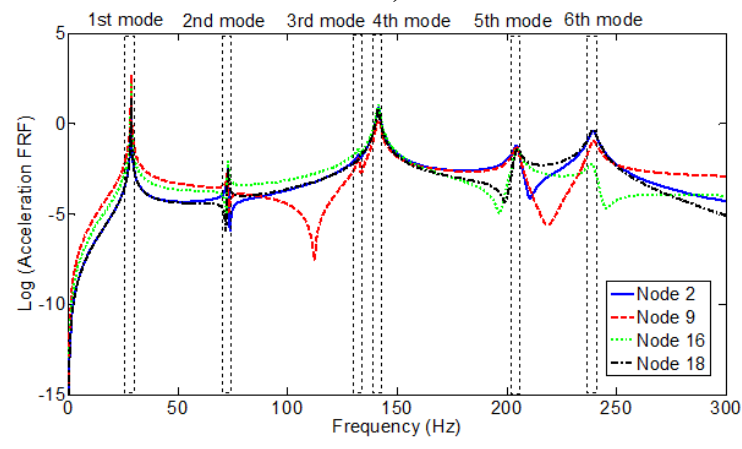

b2)

Figure 11. Comparisons of the FRF at different measured locations (4 sensors): (b1) measured locations determined based on fitness function $f_{1}$ and (b2) measured locations determined based on fitness function $f_{2}$.

4 Chow, H. M., Lam, H. F., Yin, T., et al. Optimal sensor configuration of a typical transmission tower for the purpose of structural model updating, Structural Control and Health Monitoring, 18 (3), 305-320, (2011). https://dx.doi.org/10.1002/stc.372

5 Heo, G., Wang, M. L., and Satpathi, D. Optimal transducer placement for health monitoring of long span bridge, Soil Dynamics and Earthquake Engineering, 16 (7-8), 495-502, (1997). https://dx.doi.org/10.1016/S02677261(97)00010-9

6 Cobb, R. C. and Liebst, B. S. Sensor placement and structural damage identification from minimal sensor information, AIAA Journal, 35 (2), 369-374, (1997). https://dx.doi.org/10.2514/2.103

7 Shi, Z. Y., Law, S. S., and Zhang, L. M. Optimum sensor placement for structural damage detection, Journal of Engineering Mechanics (ASCE), 126 (11), 11731179, (2000). https://dx.doi.org/10.1061/(ASCE)07339399(2000)126:11(1173) 


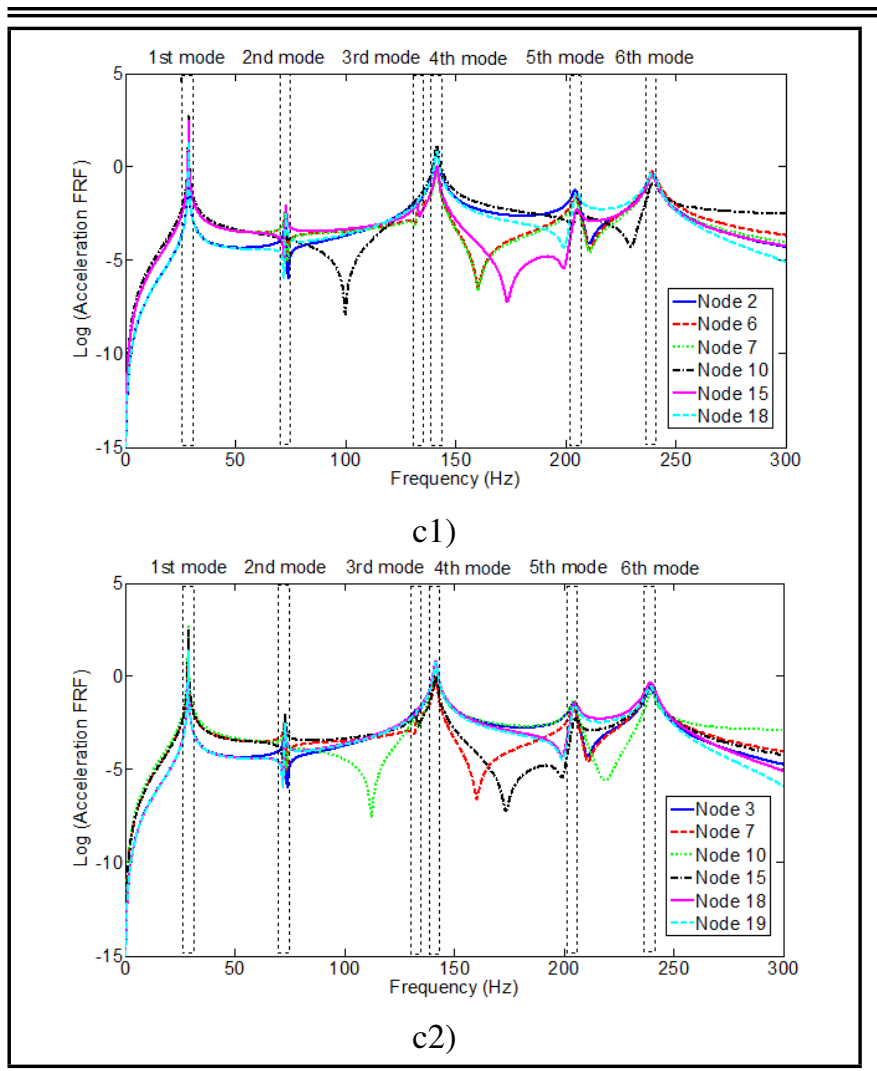

Figure 12. Comparisons of the FRF at different measured locations (6 sensors): (c1) measured locations determined based on fitness function $f_{1}$ and (c2) measured locations determined based on fitness function $f_{2}$

$8 \mathrm{Xu}, \mathrm{Z}$. D. and Wu, K. Y. Damage Detection for Space Truss Structures Based on Strain Mode under Ambient Excitation, Journal of Engineering Mechanics, 138 (10), 12151223, (2012). https://dx.doi.org/10.1061/(ASCE)EM.19437889.0000426

9 Papadimitriou, C. Optimal sensor placement methodology for parametric identification of structural systems, Journal of Sound and Vibration, 278 (4-5), 923-947, (2004). https://dx.doi.org/10.1016/j.jsv.2003.10.063

10 Papadimitriou, C. Pareto optimal sensor locations for structural identification, Computer Methods in Applied Mechanics and Engineering, 194 (12-16), 1655-1673, (2005). https://dx.doi.org/10.1016/j.cma.2004.06.043

11 Meo, M. and Zumpano, G. On the optimal sensor placement techniques for a bridge structure, Engineering Structures, 27 (10), 1488-1497, (2005). https://dx.doi.org/10.1016/j.engstruct.2005.03.015

12 Hanis, T. and Hromcik, M. Optimal sensors placement and spillover suppression, Mechanical Systems and Signal Processing, 28, 367-378, (2012). https://dx.doi.org/10.1016/j.ymssp.2011.12.007

13 Rao, A. R. M. and Anandakumar, G. Optimal placement of sensors for structural system identification and health monitoring using a hybrid swarm intelligence technique, Smart Materials and Structures, 16 (6), 2658-2672, (2007). https://dx.doi.org/10.1088/0964-1726/16/6/071

14 Yi, T. H., Li, H. N., and Zhang, X. D. A modified monkey algorithm for optimal sensor placement in struc- tural health monitoring, Smart Materials and Structures, 21 (10), 105033, (2012). https://dx.doi.org/10.1088/0964$1726 / 21 / 10 / 105033$

15 Worden, K. and Burrows, A. P. Optimal sensor placement for fault detection, Engineering Structures, 23 (8), 885-901, (2001). https://dx.doi.org/10.1016/S01410296(00)00118-8

16 Yao, L., Sethares, W. A., and Kammer, D. C. Sensor placement for on-orbit modal identification via a genetic algorithm. AIAA Journal, 31 (10), 1922-1928, (1993). https://dx.doi.org/10.2514/3.11868

17 Abdullan, M. M., Richardson, A., and Hanif, J. Placement of sensors/actuators on civil structures using genetic algorithms, Earthquake Engineering and Structural Dynamics, 30 (8), 1167-1184, (2001). https://dx.doi.org/10.1002/eqe.57

18 Guo, H. Y., Zhang, L., Zhang, L. L., et al. Optimal placement of sensors for structural health monitoring using improved genetic algorithms, Smart Materials and Structures, 13 (3), 528-534, (2004). https://dx.doi.org/10.1088/0964$1726 / 13 / 3 / 011$

19 Liu, W., Gao, W. C., Sun, Y., et al. Optimal sensor placement for spatial lattice structure based on genetic algorithms, Journal of Sound and Vibration, 317 (1-2), 175189, (2008). https://dx.doi.org/10.1016/j.jsv.2008.03.026

${ }^{20}$ Kennedy, J. and Eberhart, R. Particle swarm optimisation, In: Proceedings of the IEEE International Conference on Neural Networks, Perth, Australia, 1942-1948, (1995). https://dx.doi.org/10.1109/ICNN.1995.488968

21 Li, Z. N., Tang, J., and Li, Q. S. Optimal sensor locations for structural vibration measurements, Applied Acoustics, 65 (8), 807-818, (2004). https://dx.doi.org/10.1016/j.apacoust.2003.12.007

22 Allemang, R. J. and Brown, D. L. A correlation coefficient for modal vector analysis, In: Proceedings of the First International Modal Analysis Conference, Orlando, FL, 110116, (1982).

$23 \mathrm{Ha}, \mathrm{A}$. and Liu, L. Sensor and actuator location in motion control of flexible structures, Journal of Sound and Vibration, 167 (2), 239-261, (1993). https://dx.doi.org/10.1006/jsvi.1993.1333

24 Nimityongskul, S. and Kammer, D. C. Frequency response based sensor placement for the mid-frequency range, Mechanical Systems and Signal Processing, 23 (4), 1169-1179, (2009). https://dx.doi.org/10.1016/j.ymssp.2008.11.006

25 Lin, C. S. and Chiang, D. Y. A modified random decrement technique for modal identification from nonstationary ambient response data only, Journal of Mechanical Science and Technology, 26 (6), 1687-1696, (2012). https://dx.doi.org/10.1007/s12206-012-0920-7 